\title{
Avaliação de extratos de macroalgas bênticas do litoral catarinense utilizando o teste de letalidade para Artemia salina
}

\author{
Cintia Lhullier', Paulo Antunes Horta ${ }^{2}$, Miriam Falkenberg ${ }^{3 *}$ \\ ${ }^{1}$ Programa de Pós-Graduação em Farmácia, Centro de Ciências da Saúde, Universidade Federal de Santa \\ Catarina, 88040-970, Florianópolis, SC, Brasil, \\ ${ }^{2}$ Departamento de Botânica, Centro de Ciências da Saúde, Universidade Federal de Santa Catarina, \\ 88040-970, Florianópolis, SC, Brasil, \\ ${ }^{3}$ Departamento de Ciências Farmacêuticas, Centro de Ciências da Saúde, Universidade Federal de Santa Catarina, \\ 88040-970, Florianópolis, SC, Brasil
}

\begin{abstract}
RESUMO: Foram coletadas amostras de algas marinhas de 19 espécies (sendo 4 pertencentes ao filo Chlorophyta, 5 ao filo Phaeophyta e 10 ao filo Rhodophyta) em dois locais do litoral catarinense. Os extratos etanólicos foram submetidos ao teste de letalidade para larvas de Artemia salina com objetivo de realizar uma triagem das espécies. Dos 26 extratos testados, 25 apresentaram toxicidade significativa em pelo menos uma das 3 concentrações testadas. O grupo de algas vermelhas (Rhodophyta) foi o que obteve maior porcentagem de extratos com resultados estatisticamente significativos pelo método do qui-quadrado e também menores valores de $\mathrm{CL}_{50}$, com destaque para Acanthophora spicifera, Hypnea musciformis e Pterocladiella capillacea. Observaram-se diferenças entre as espécies de um mesmo gênero (Codium decorticatum e Codium isthmocladium) e também a influência de fatores ambientais (Hypnea musciformis) na toxicidade dos extratos.
\end{abstract}

Unitermos: Artemia salina, macroalgas, toxicidade, litoral catarinense.

\begin{abstract}
Evaluation of macroalgae from Santa Catarina's coast with the brine shrimp assay". Samples of 19 macroalgae species (4 Chlorophyta, 5 Phaeophyta and 10 Rhodophyta) have been collected from two points of Santa Catarina's coast. The ethanolic extracts were avaluated with the brine shrimp assay in order to perform a screening for potential toxicity. A total of 25 extracts presented significant results in one or more of the tested concentrations. The phylum Rhodophyta presented more statistically significant results with the chi-square test, as well as lower values of $\mathrm{LC}_{50}$. The extracts of Acanthophora spicifera (from Canasvieiras and Ilha do Francês), Hypnea musciformis and Pterocladiella capillacea (both from Ilha do Francês) presented $\mathrm{LC}_{50}$ below $50 \mu \mathrm{g} / \mathrm{mL}$. Differences between the species of same genus (Codium decorticatum and Codium isthmocladium) and the influence of environmental factors (Hypnea musciformis) were observed.
\end{abstract}

Keywords: Artemia salina, macroalgae, brine shrimp assay, Santa Catarina's coast

\section{INTRODUÇÃO}

Algas são organismos eucariotos que possuem clorofila a e um talo não diferenciado em raiz, caule ou folhas, com hábito predominantemente aquático. Neste ambiente, as algas podem fazer parte dos bentos (indivíduos fixos no substrato) ou plâncton (indivíduos suspensos na água). O litoral do estado de Santa Catarina corresponde à região Temperada Quente e apresenta flora rica em número de espécies, havendo um predomínio de espécies de algas vermelhas (Oliveira Filho, 1977).

Compostos bioativos são quase sempre tóxicos em altas doses. Desta maneira, a avaliação da letalidade em um organismo animal menos complexo pode ser usada para um monitoramento simples e rápido durante o fracionamento de extratos. O ensaio de letalidade para larvas de Artemia salina tem sido introduzido na rotina de muitos grupos de pesquisa envolvidos com isolamento, purificação e elucidação estrutural, já que muitos laboratórios de fitoquímica não estão preparados para a realização de ensaios biológicos (McLaughlin, 1991; Falkenberg; Baumgarten; Simionato, 1999; Maciel; Pinto; Veiga Jr., 2002; Ruiz et al., 2005). Os cistos de Artemia salina são de baixo custo e facilmente encontrados no comércio, além de permanecerem viáveis por anos no estado seco (Meyer et al., 1982). Essas vantagens contribuíram para a popularização do bioensaio, sobretudo a partir da década de 90 .

O ensaio foi proposto inicialmente por Michael, Thompson e Abramovitz (1956), e posteriormente desenvolvido por Vanhaecke et al. (1981), bem como por Sleet e Brendel (1983), baseando-se na possibilidade de imobilizar náuplios de Artemia salina em culturas laboratoriais (Carballo et al., 2002). A metodologia admite variações. Solis et al. (1993) propõem a realização deste ensaio em microplacas ao invés de tubos e sugerem 
a utilização do ensaio também na triagem de novos fármacos antiespasmódicos e antimaláricos.

Esta metodologia tem sido empregada ainda para detectar toxicidade preliminar de algas marinhas (Ara et al., 1999), realizar triagem de toxinas fúngicas (Harwig; Scott, 1971), avaliar efeitos de exposição a metais pesados (Martinez et al., 1999) e pesticidas (Barahona; SánchezFortún, 1999) e para testes de toxicidade em materiais dentários (Pelka et al., 2000).

Carballo et al. (2002) compararam extratos de produtos marinhos com o ensaio de letalidade com larvas de $A$. salina e quanto à citotoxicidade em 2 linhagens de células humanas. Segundo os autores, os resultados apresentam uma boa correlação, tal como já estabelecido para extratos de plantas (McLaughlin, 1991), sugerindo que este bioensaio seja utilizado para testar produtos naturais marinhos com potencial atividade farmacológica.

Considerando os resultados interessantes obtidos em estudos anteriores e a escassez de trabalhos semelhantes com espécies de algas brasileiras, propusemos a realização de uma triagem de macroalgas bênticas do estado de Santa Catarina biomonitorada pelo ensaio de letalidade utilizando larvas de Artemia salina, abrangendo espécies de algas vermelhas (Rhodophyta), pardas (Phaeophyta) e verdes (Chlorophyta).

\section{MATERIAL E MÉTODOS}

\section{Coleta do Material}

Amostras de macroalgas bênticas foram coletadas nas praias de Canasvieiras e Ilha do Francês, na faixa de médio e infra-litoral do estado de Santa Catarina, Brasil, no período de novembro a dezembro de 2002. A identificação do material foi feita pelo Prof. Dr. Paulo Antunes Horta (UFPb).

\section{Preparação dos extratos}

Após a coleta, as algas foram armazenadas em etanol (EtOH) 99\% para transporte. Procedeu-se à limpeza, com a retirada de todas as epífitas, lavagem com água destilada, e secagem por 7 dias à temperatura ambiente $\left(25^{\circ} \mathrm{C}\right)$, dentro de estufa. Em seguida, o material foi rasurado e macerado em EtOH 99\% por 7 dias, na proporção $1: 10(\mathrm{~m} / \mathrm{V})$, incluindo o solvente utilizado no transporte da respectiva alga. $\mathrm{O}$ extrato foi filtrado e concentrado em rotavapor sob temperatura inferior a 60 ${ }^{\circ} \mathrm{C}$ até secura total dos extratos.

\section{Bioensaio de letalidade para larvas de Artemia salina}

Foi utilizada a metodologia descrita por Meyer et al. (1982) com algumas modificações. Os cistos de Artemia salina foram colocados em um aquário com água do mar sob aeração e controle da temperatura $\left(20-30{ }^{\circ} \mathrm{C}\right)$ e após $48 \mathrm{~h}$ de incubação, as larvas foram retiradas para o ensaio.

\section{Preparação das diluições seriadas dos extratos}

Foram realizadas diluições seriadas dos extratos de macroalgas bênticas com EtOH 99\% em tubos de ensaio, de acordo com o cálculo do rendimento, de modo a possibilitar a obtenção das concentrações finais de $200 \mu \mathrm{g} / \mathrm{mL}, 100 \mu \mathrm{g} / \mathrm{mL}$ e $50 \mu \mathrm{g} / \mathrm{mL}$. As soluções dos extratos foram aquecidas em banho-maria $\left(50{ }^{\circ} \mathrm{C}\right)$ até total evaporação do solvente. Foram preparados tuboscontrole contendo somente solvente ao invés de extrato. Para cada extrato de alga foram preparadas 3 séries de tubos (uma série para cada concentração, com 3 tubos de ensaio em cada série).

\section{Incubação das larvas com o material}

A todos os tubos de ensaio foram adicionados $1,0 \mathrm{~mL}$ de água do mar e $50 \mu \mathrm{L}$ de dimetilsulfóxido. Os tubos foram colocados em sonicador por $10 \mathrm{~min}$ e, com auxílio de uma pipeta graduada, foram transferidas 10 larvas para cada tubo de ensaio. O volume do tubo foi completado com água do mar para $5 \mathrm{~mL}$.

\section{Contagem das larvas}

Após $24 \mathrm{~h}$ em contato com a suspensão dos extratos, realizou-se a contagem do número de larvas sobreviventes. Foram consideradas mortas aquelas larvas que permaneceram imóveis por mais de 10 segundos após agitação suave dos tubos.

\section{Análise estatística dos resultados}

O cálculo da concentração letal média $\left(\mathrm{CL}_{50}\right)$ dos extratos foi feito a partir das três concentrações estudadas utilizando o programa PROBIT. exe (disponível em www. probit.net).

A análise estatística dos dados do ensaio de $A$. salina também foi feita pelo teste de qui-quadrado, com tabela de contingência $2 \times 2$, utilizando a correção de Yates, sendo considerados significativos $(\alpha=0,05)$ os valores de $\chi^{2}$ maiores ou iguais a 3,84 (Centeno, 1990).

\section{RESULTADOS E DISCUSSÃO}

\section{Cálculo da $\mathrm{CL}_{50}$}

No presente estudo, 12 de 26 extratos apresentaram valores de $\mathrm{CL}_{50}$ inferiores a $50 \mu \mathrm{g} / \mathrm{mL}$, sendo 7 extratos de algas vermelhas, 3 de algas pardas e 2 de algas verdes (Tabela 1). O predomínio de baixos valores de $\mathrm{CL}_{50}$ entre os extratos de algas pertencentes ao filo Rhodophyta poderia ser explicado, ao menos em parte, pela relativa semelhança entre os filos Cyanophyta 
Tabela 1. Valores de $\mathrm{CL}_{50}$ calculados para os diferentes extratos de macroalgas bênticas; valores entre parênteses correspondem ao intervalo de confiança $(\mathrm{F}=$ Ilha do Francês e $\mathrm{C}=$ Canasvieiras $)$.

\begin{tabular}{|c|c|c|}
\hline Filo & Alga marinha & $\mathrm{CL}_{50}(\mu \mathrm{g} / \mathrm{mL})$ \\
\hline \multirow{12}{*}{ Rhodophyta } & Acanthophora spicifera $\mathrm{F}$ & $<50$ \\
\hline & Acanthophora spicifera $\mathrm{C}$ & $<50$ \\
\hline & Bostrychia sp C & $52,8(31,144-68,561)$ \\
\hline & Centroceras clavulatum $\mathrm{C}$ & $<50$ \\
\hline & Chondracanthus acicularis $\mathrm{C}$ & $53,4(36,847-65,756)$ \\
\hline & Galaxaura marginata $\mathrm{F}$ & $<50$ \\
\hline & Gracilaria dominguensis $\mathrm{C}$ & $<50$ \\
\hline & Grateloupia doryphora $\mathrm{C}$ & $57,8(12,217-88,531)$ \\
\hline & Hypnea musciformis $\mathrm{F}$ & $<50$ \\
\hline & Hypnea musciformis $\mathrm{C}$ & $97,5(68,524-128,814)$ \\
\hline & Laurencia sp F & $85,0(65,647-100,342)$ \\
\hline & Pterocladiella capillacea $\mathrm{F}$ & $<50$ \\
\hline \multirow[t]{8}{*}{ Phaeophyta } & Colpomenia sinuosa $\mathrm{F}$ & $79,4(50,150-106,721)$ \\
\hline & Dictyota cervicomis F & $51,0(27,076-67,877)$ \\
\hline & Ectocarpus breviarticulatus $\mathrm{C}$ & $<50$ \\
\hline & Padina gymnospora $\mathrm{F}$ & $<50$ \\
\hline & Padina gymnospora $\mathrm{C}$ & $<50$ \\
\hline & Sargassum stenophyllum $\mathrm{F}$ & $88,5(65,847-110,150)$ \\
\hline & Sargassum stenophyllum C & $98,1(71,581-125,140)$ \\
\hline & Sargassum vulgare & $67,4(35,348-92,955)$ \\
\hline \multirow[t]{6}{*}{ Chlorophyta } & Chaetomorpha antennina $\mathrm{C}$ & $<50$ \\
\hline & Codium decorticatum $\mathrm{C}$ & $<50$ \\
\hline & Codium isthmocladium $\mathrm{F}$ & $229,2(144,459-2459,067)$ \\
\hline & Enteromorpha intestinalis $\mathrm{C}$ & $57,1(31,073-76,497)$ \\
\hline & Ulva fasciata $\mathrm{F}$ & $76,2(45,432-103,535)$ \\
\hline & Ulva fasciata $\mathrm{C}$ & $63,2(22,556-92,743)$ \\
\hline
\end{tabular}

(algas azuis ou cianobactérias) e Rhodophyta (Horta, 2000). Em estudo realizado por Falch et al. (1995), extratos etanólicos de cianobactérias apresentaram uma elevada toxicidade para Artemia salina, com $\mathrm{CL}_{50}$ inferior a $50 \mu \mathrm{g} / \mathrm{mL}$.

Além dos resultados mais destacados entre as algas vermelhas, a tabela 1 evidencia toxicidades altas em extratos de algas pardas (Phaeophyta), como Ectocarpus breviarticulatus $\left(\mathrm{CL}_{50}<50 \mu \mathrm{g} / \mathrm{mL}\right)$ e Padina gymnospora $\left(\mathrm{CL}_{50}<50 \mu \mathrm{g} / \mathrm{mL}\right)$. Ara et al. (1999) avaliaram pelo ensaio de letalidade para larvas de Artemia salina 22 extratos etanólicos de macroalgas bênticas (13 algas pardas, 6 algas verdes e 3 algas vermelhas) provenientes da costa de Karachi, sendo que apenas 6 extratos apresentaram $\mathrm{CL}_{50}$ abaixo de $50 \mu \mathrm{g} / \mathrm{mL}$. Desses, 5 eram provenientes de algas pardas, levando aqueles pesquisadores a sugerir que extratos etanólicos provenientes de algas pardas eram os mais tóxicos, quando comparadas as suas concentrações letais médias com aquelas de extratos de algas verdes e vermelhas. No trabalho que deu origem a este artigo (Lhullier, 2005) quase 50\% das amostras eram de espécies de algas vermelhas. Assim, evidencia-se a potencial influência do percentual relativo de espécies testadas de cada filo nos resultados obtidos (Tabela 2).
Entretanto, em relação ao filo Rhodophyta , cabe destacar que o mesmo possui diferenças em relação às outras algas eucarióticas, como ausência de estágios flagelados, presença de ficobilinas e clorofila d, tilacóides não agregados nos cloroplastos e reprodução sexuada oogâmica envolvendo células femininas (carpogônio) e masculinas (espermácio). Estas características poderiam estar associadas a um maior estágio evolutivo e conseqüentemente à produção de metabólitos secundários diversificados (Van Den Hoek; Mann; Jahns, 1995).

A análise comparativa da $\mathrm{CL}_{50}$ evidencia ainda diferenças na atividade tóxica entre espécies distintas de um gênero de Chlorophyta: Codium decorticatum, com $\mathrm{CL}_{50}$ inferior a $50 \mu \mathrm{g} / \mathrm{mL}$ e Codium isthmocladium, com $\mathrm{CL}_{50}^{50}$ igual $229,2 \mu \mathrm{g} / \mathrm{mL}$.

Para os extratos da espécie Hypnea musciformis (Rhodophyta) foram observadas diferenças na $\mathrm{CL}_{50}$ em função dos locais de coleta (Ilha do Francês: $\mathrm{CL}_{50}$ $<50 \mu \mathrm{g} / \mathrm{mL}$; Canasvieiras: $\mathrm{CL}_{50}=97,5 \mu \mathrm{g} / \mathrm{mL}$ ). Neste caso, é possível que fatores como o hidrodinamismo (Domínguez-Bocanegra et al., 2004), herbivoria (Alcoverro; Duarte; Romero, 1997), salinidade (Wraige et al., 1998) e/ou pH (Horta et al., 2001) estejam influenciando a produção de metabólitos secundários das 
Tabela 2. Comparação dos números de espécies de algas marinhas testadas para cada filo nos trabalhos desenvolvidos por Ara et al. (1999) e Lhullier (2005) em relação ao número de extratos com valores de $\mathrm{CL}_{50}<50 \mu \mathrm{g} / \mathrm{mL}$.

\begin{tabular}{cccc}
\hline \multicolumn{3}{c}{$\mathrm{N}^{\circ}$ de amostras testadas $\left(\mathrm{n}^{\circ}\right.$ de extratos com $\left.\mathrm{CL}_{50}<50 \mu \mathrm{g} / \mathrm{mL}\right)$} \\
\hline & Chlorophyta & Phaeophyta & Rhodophyta \\
Ara et al. (1999) & $6(0)$ & $13(5)$ & $3(1)$ \\
Lhullier (2005) & $6(2)$ & $8(3)$ & $12(7)$ \\
\hline
\end{tabular}

Tabela 3. Valores de $\chi^{2}$ calculados para os diferentes extratos de macroalgas bênticas no ensaio de letalidade para larvas de $A$. salina. $(\mathrm{F}=$ Ilha do Francês e $\mathrm{C}=$ Canasvieiras).

\begin{tabular}{|c|c|c|c|c|}
\hline Filo & Alga marinha & $50 \mu \mathrm{g} / \mathrm{mL}$ & $100 \mu \mathrm{g} / \mathrm{mL}$ & $200 \mu \mathrm{g} / \mathrm{ml}$ \\
\hline \multirow[t]{12}{*}{ Rhodophyta } & Acanthophora spicifera $\mathrm{F}$ & $5,75^{*}$ & $10,93 *$ & $18,03 *$ \\
\hline & Acanthophora spicifera $\mathrm{C}$ & 0,96 & $8,37 *$ & $18,03 *$ \\
\hline & Bostrychia sp C & 2,99 & $8,37 *$ & $18,03 *$ \\
\hline & Centroceras clavulatum $\mathrm{C}$ & $6,95^{*}$ & $10,11^{*}$ & $18,03^{*}$ \\
\hline & Chondracanthus acicularis $\mathrm{C}$ & 2,99 & $10,11^{*}$ & $18,03 *$ \\
\hline & Galaxaura marginata $\mathrm{F}$ & 1,26 & $8,37 *$ & $18,03 *$ \\
\hline & Gracilaria dominguensis $\mathrm{C}$ & 0,96 & 3,18 & $8,37 *$ \\
\hline & Grateloupia doryphora $\mathrm{C}$ & 0,08 & 0,71 & $10,93 *$ \\
\hline & Hypnea musciformis $\mathrm{F}$ & $5,75^{*}$ & $10,93 *$ & $18,03 *$ \\
\hline & Hypnea musciformis $\mathrm{C}$ & 0,04 & 3,18 & $22,16^{*}$ \\
\hline & Laurencia sp F & 3,18 & $8,37 *$ & $14,08 *$ \\
\hline & Pterocladiella capillacea $\mathrm{F}$ & 0,71 & 2,06 & $10,93 *$ \\
\hline \multirow[t]{8}{*}{ Phaeophyta } & Colpomenia sinuosa $\mathrm{F}$ & 0,23 & 2,06 & $10,11 *$ \\
\hline & Dictyota cervicomis F & 1,26 & $6,95 *$ & $18,03 *$ \\
\hline & Ectocarpus breviarticulatus $\mathrm{C}$ & 0,51 & $8,37 *$ & $10,93 *$ \\
\hline & Padina gymnospora $\mathrm{F}$ & 1,65 & $6,95 *$ & $14,80 *$ \\
\hline & Padina gymnospora $\mathrm{C}$ & $4,72 *$ & $10,11 *$ & $18,03 *$ \\
\hline & Sargassum stenophyllum $\mathrm{F}$ & 0,05 & 0,71 & $10,93 *$ \\
\hline & Sargassum stenophyllum $\mathrm{C}$ & 0,96 & 2,06 & $10,11 *$ \\
\hline & Sargassum vulgare & 0,08 & 0,96 & $18,03 *$ \\
\hline \multirow[t]{6}{*}{ Chlorophyta } & Chaetomorpha antennina $\mathrm{C}$ & $6,95 *$ & $10,11 *$ & $18,03 *$ \\
\hline & Codium decorticatum $\mathrm{C}$ & 2,99 & $10,11 *$ & $18,03 *$ \\
\hline & Codium isthmocladium $\mathrm{F}$ & 0,04 & 0,05 & 0,96 \\
\hline & Enteromorpha intestinalis $\mathrm{C}$ & $8,37 *$ & $10,93 *$ & $22,16^{*}$ \\
\hline & Ulva fasciata $\mathrm{F}$ & 0,96 & 2,06 & $10,11 *$ \\
\hline & Ulva fasciata $\mathrm{C}$ & 0,51 & 1,26 & $10,93 *$ \\
\hline
\end{tabular}

*Resultados $\geq 3,84$ considerados significativos $(\alpha=0,05)$.

algas. Além destes aspectos, também a sazonalidade pode vir a ser um fator determinante para a atividade biológica de extratos de algas, como demonstrado por Imbs et al. (2001), que evidenciaram aumento na produção de prostaglandinas na alga vermelha Gracilaria verrucosa nos meses de menor radiação solar.

\section{Análise estatística pelo método do qui-quadrado}

Para os extratos cuja $\mathrm{CL}_{50}$, em princípio, só poderia ser obtida por extrapolação dos resultados, por ser inferior a $50 \mu \mathrm{g} / \mathrm{mL}$ (a concentração mais baixa testada) recorreu-se ao teste do qui-quadrado como alternativa para a análise destes resultados (Tabela 3). Do total de 26 extratos testados, 25 apresentaram toxicidade na maior concentração testada. Considerando-seas 3 concentrações, $64 \%$ dos extratos testados de algas vermelhas, 56\% de algas verdes e $54 \%$ de algas pardas apresentaram toxicidade significativa. Pelos dados apresentados observa-se um predomínio de resultados significativos em extratos de algas marinhas vermelhas (Rhodophyta), como Acanthophora spicifera, Centroceras clavulatum e Hypnea musciformis (Ilha do Francês). Em relação às outras classes de algas marinhas, no filo de algas pardas (Phaeophyta), destaca-se Padina gymnospora e, nas algas verdes (Chlorophyta), Enteromorpha intestinalis e Chaetomorpha antennina, que apresentaram resultados estatisticamente significativos nas 3 concentrações avaliadas.

$\mathrm{Na}$ literatura pesquisada não foram encontrados relatos de testes de substâncias isoladas de algas com o bioensaio de Artemia salina. Entretanto, as algas vermelhas (Rhodophyta) são reconhecidas como as maiores produtoras de substâncias halogenadas no meio marinho (Fenical, 1975; Teixeira; Kelecom; Gottlieb, 1991). Dentre elas, o gênero Laurencia Lamouroux 
destaca-se como uma fonte fascinante de novos produtos naturais (Erickson, 1983), sendo o gênero de algas marinhas mais investigado.

Em relação às atividades relatadas para extratos e substâncias isoladas de espécies de Laurencia, em estudos realizados por Iliopoulou et al. (2002) metabólitos isolados de Laurencia obtusa apresentaram atividade contra HIV-1 ( III $\left._{\mathrm{B}}\right), \mathrm{HIV}-2$ (ROD), vírus de estomatite vesicular, Coxsackie B4, vírus respiratório sincicial, entre outros vírus.

Estudos realizados por Juagdan, Kalidindi e Scheuer (1997) com metabólitos halogenados de Laurencia cartilaginea revelam atividade citotóxica para linhagens celulares cancerígenas P-388, A-549, MEL28 e especialmente HT-29 (carcinoma de cólon). Já para diterpenos halogenados de Laurencia obtusa foi detectada atividade citotóxica para as linhagens celulares MCF7 (derivada do adenocarcinoma mamário), PC3 (derivada do adenocarcinoma prostático), entre outras (Iliopoulou et al., 2003). Também foi encontrado potencial citotóxico para sesquiterpenos de Laurencia tristicha contra várias linhagens de células tumorais, como HeLa (Sun et al., 2005), enquanto El-Gamal, Wang e Duh (2005) comprovaram citotoxicidade de polibrometos sulfatados de Laurencia brongniartii em linhagens como HT-29.

A tabela 3 também evidencia diferenças entre espécies de um mesmo gênero, como Codium decorticatum e Codium isthmocladium (Chlorophyta), que não foram encontradas no mesmo local. Para a primeira, foram obtidos índices de letalidade estatisticamente significativos nas concentrações de 100 e $200 \mu \mathrm{g} / \mathrm{mL}$, enquanto o extrato de Codium isthmocladium não apresentou toxicidade significativa em nenhuma das concentrações testadas. Estes resultados poderiam ser atribuídos às diferenças propriamente ditas entre as espécies, como ao fato de terem sido coletadas em locais diferentes (fatores ambientais). Para as 3 amostras do gênero Sargassum (Phaeophyta), que incluem 2 espécies distintas coletadas no mesmo local, só foram obtidos índices de letalidade significativos na concentração mais alta. O extrato de $S$. stenophyllum da Ilha do Francês na concentração de $200 \mu \mathrm{g} / \mathrm{mL}$ apresentou toxicidade inferior à de $S$. vulgare do mesmo local de coleta, mas superior à do extrato de $S$. stenophyllum de Canasvieiras.

A análise por este método também permite observar a relevância da influência do local de coleta na diferença dos índices dos extratos de Hypnea musciformis (Rhodophyta) coletada na praia de Canasvieiras (dados estatisticamente significativos apenas na concentração $200 \mu \mathrm{g} / \mathrm{mL}$ ) e a da mesma espécie coletada na Ilha do Francês (dados estatisticamente significativos nas 3 concentrações estudadas), confirmando a influência dos fatores ambientais na produção de metabólitos por macroalgas bênticas.

\section{REFERÊNCIAS}

Alcoverro T, Duarte CM, Romero J 1997. The influence of herbivores on Posidonia oceanica epiphytes. Aquat Bot 56: 93-104.

Ara J, Sultana V, Ehteshamul-Haque S, Qasim R, Ahmad VU 1999. Cytotoxic activity of marine macro-algae on Artemia salina (brine shrimp). Phytother Res 13: 304 307.

Barahona MV, Sánchez-Fortún S 1999. Toxicity of carbamates to the brine shrimp Artemia salina and the effect of atropine, BW284c51, iso-OMPA and 2-PAM on carbaryl toxicity. Environ Pollut 104: 469-476.

Carballo JL, Hernández-Inda ZL, Pérez P, García-Grávalos MD 2002. A comparison between two brine shrimp assays to detect in vitro cytotoxicity in marine natural products. BMC Biotechnol 2: 1-5.

Centeno AJ 1990. Curso de estatística aplicada à biologia. Goiânia: Cegraf-UFG.

Domínguez-Bocanegra AR, Legarreta IG, Jeronimo FM, Campocosio AT 2004. Influence of environmental and nutritional factors in the production of astaxanthin from Haematococcus pluvialis. Bioresour Technol 92: 209-214.

El Gamal AA, Wang W, Duh C 2005. Sulfur-containing polybromoindoles from the formosan red alga Laurencia brongniartii. J Nat Prod 68: 815-817.

Erickson KL 1983. In: Scheuer, PJ (ed.) Marine natural products. chemical and biological perspectives. New York: Academic Press, p.131-257.

Falch BS, König GM, Wright AD, Sticher O, Angerhofer CK, Pezzuto JM, Bachmann H 1995. Biological activities of cyanobacteria: evaluation of extracts and pure compounds. Planta Med 61: 321-328.

Falkenberg M, Baumgarten D, Simionato C 1999. Screening of some Brazilian medicinal plants with the brine shrimp assay. Acta Hortic 502: 401-404.

Fenical W 1975. Halogenation in the Rhodophyta: a review. $J$ Phycol 11: 245-259.

Harwig J, Scott P 1971. Brine shrimp (Artemia salina) larvae as a screening system for fungal toxins. Appl Environ Microbiol 21: 1011-1016.

Horta PA 2000. Aspectos taxonomicos e ecologia de macroalgas bênticas. Florianópolis:UFSC.

Horta PA, Amancio E, Coimbra CS, Oliveira EC 2001. Considerações sobre a distribuição e origem da flora de macroalgas brasileiras. Hoehnea 28: 243-265.

Iliopoulou D, Roussis V, Pannecouque C, De Clercq E, Vagias C 2002. Halogenated sesquiterpenes from the red alga Laurencia obtusa. Tetrahedron 58: 6749-6755.

Iliopoulou D, Mihopoulos N, Vagias C, Papazafiri P, Roussis V 2003. Novel cytotoxic brominated diterpenes from the red alga Laurencia obtusa. J Org Chem 68: 76677674.

Imbs AB, Vologodskaya AV, Nevshupova NV, Khotimchenko SV, Tilyanov EA 2001. Response of prostaglandin content in the red alga Gracilaria verrucosa to season and solar irradiance. Phytochemistry 58: 1067-1072.

Juagdan EG, Kalidindi R, Scheuer P 1997. Two new chamigranes from a Hawaiian red alga, Laurencia cartilaginea. Tetrahedron 53: 521-528.

Lhullier C 2005. Triagem de macroalgas bênticas do litoral de Santa Catarina biomonitorada pelo ensaio de 
letalidade para larvas de Artemia salina e investigação fitoquímicade Pterocladiella capillacea. Florianópolis, 100p. Dissertação de Mestrado - Programa de PósGraduação em Farmácia, Universidade Federal de Santa Catarina.

Maciel MAM, Pinto AC, Veiga Jr VF 2002. Plantas Medicinais: a necessidade de estudos interdisciplinares. Quim Nova 25: 429-438.

Martinez M, Del Ramo J, Torreblanca A, D'iaz-Mayans J 1999. Effect of cadmium exposure on zinc levels in the brine shrimp Artemia partenogenetics. Aquaculture 172: 315-325.

McLaughlin JL 1991. Crown gall tumours on potato discs and brine shrimp lethality: two simple bioassays for higher plant screening and fractions. In: Dey PM, Harbone JB (ed.) Methods in Plant Biochemistry. New York: Academic Press, p.1-32.

Meyer BN, Ferrigni NR, Putnam JE, Jacobsen LB, Nichols DE, McLaughlin JL 1982. Brine shrimp: a convenient general bioassay for active plant constituents. Planta Med 45: 31-34.

Michael AS, Thompson CG, Abramovitz M 1956. Artemia salina as a test organism for a bioassay. Science 123: 464-468.

Oliveira-Filho EC 1977. Algas marinhas bentônicas do Brasil. São Paulo, 407p. Tese de Doutorado - Universidade de São Paulo.

Pelka M, Danzl C, Distler W, Petschelt A 2000. A new screening test for toxicity testing of dental materials. J Dent 28:341-345.

Ruiz ALTG, Magalhães EG, Magalhães AF, Faria AD, Amaral MCE, Serrano DR, Zanotti-Magalhães EM, Magalhães LA 2005 Avaliação da atividade tóxica em Artemia salina e Biomphalaria glabrata de extratos de quatro espécies do gênero Eleocharis (Cyperaceae). Rev Bras Farmacogn 15: 98-102.

Sleet RB, Brendel K 1983. Improved methods for harvesting and counting synchronous populations of Artemia nauplii for use in developmental toxicology. Ecotoxicol Environ Saf 7: 435-446.

Solis PN, Wright CW, Anderson MM, Gupta MP, Phillipson JD 1993. A microwell cytotoxicity assay using Artemia salina. Planta Med 59: 250-252.

Sun J, Shi D, Ma M, Li S, Wang S, Han L, Yang Y, Fan X, Shi J, He L 2005. Sesquiterpenes from the red alga Laurencia tristicha. J Nat Prod 68: 915-919.

Teixeira VL, Kelecom A, Gottlieb OR 1991. Produtos naturais de algas marinhas. Quim Nova 14: 83-90.

Van Den Hoek C, Mann DG, Jahns HM 1995. Algae: an introduction to phycology. Cambridge: Academic Press.

Vanhaecke P, Persoone G, Claus C, Sorgeloos P 1981. Proposal for a short-term toxicity test with Artemia nauplii. Ecotoxicol Environ Saf 5: 382-387.

Wraige EJ, Belt ST, Massé G, Robert JM, Rowland SJ 1998. Variations in distributions of $\mathrm{C}_{25}$ highly branched isoprenoid (HBI) alkenes in the diatom, Haslea ostrearia: influence of salinity. Org Geochem 28: 855859. 